Proceedings of Magneto-Optical Recording International Symposium '92, J. Magn. Soc. Jpn., Vol. 17, Supplement No. S1 (1993), pp. 213-214 (1) 1993 by The Magnetics Society of Japan

\title{
WRITE PERFORMANCE AND THE INFLUENCE OF BIAS POWER
}

\author{
B. I. Finkelstein \\ Optical Integration \\ ADSTAR, IBM Tucson
}

\author{
G. J. Wagner \\ Optical Integration \\ ADSTAR, IBH Tucson
}

INTRODUCTION - Future optical storage systems will involve higher linear densities, track densities, and data rates; however, increasing storage capacity and data rate will reduce drive analog performance margins. As a result, a drive should be designed to obtain maximum performance from the disk to maintain data integrity. A disk conforming to the proposed ECMA Extended Capacity standard (1163 b/mm, 1.39 um track pitch) was used to investigate the interaction between bias power (or pedestal power), write power, and data rate. For experimental purposes, $2400 \mathrm{rpm}$ were used. This paper explores the effects of bias power (the power from which the laser write pulse starts) on signal amplitude, resolution, jitter, peak shift, and data reliability figure-of-merit (FOM)

THE EXPERIMENT - Tests were conducted at four different bias powers $(0.5,1.0,1.5$, and $2.0 \mathrm{~mW})$ and at disk velocities of 7.54 and $14.61 \mathrm{mps}$ (same linear density). The $14.61 \mathrm{mps}$ case is equivalent to the ID track of the $O D$ band, where the system experiences simultaneously the highest data rate and linear density. The $7.54 \mathrm{mps}$ case represents the velocity at the ID track of the ID band. which is the slowest velocity (and data rate) that can occur, and it is most likely to experience adverse thermal effects. Data was obtained for several quantities as a function of bias power and write power that ranged from 5.0 to $14.5 \mathrm{~mW}$. The high density pattern (3T), the low density pattern (8T), and a complex pattern, were written separately and signal amplitude and jitter were measured. The complex pattern was the asymmetric " $M$ " paltern (AM pattern - 7T 3T $8 T$ ) that allowed the measurement of peak shift.

ANALYSIS/RESULTS - Measurements of signal amplitude show that for a given write power the 8T amplitude increases as bias power increases until saturation occurs at an amplitude of about $0.45 \mathrm{~V}$. At low write powers, the 3T amplitude increases as bias power increases. but at high write powers the amplitude decreases as bias power increases (Figures 1 and 2). Usually. signal amplitude depends on pattern, write power, bias power, and data rate. However, if the point of highest $3 T$ amplitude is used to determine the optimum write power for each bias power, then $3 T$ and $8 T$ amplitude is independent of bias power and velocity, provided this power is used when writing.

As bias power increases, the optimum write power decreases because of the higher media temperature before the write pulse. When a write pulse occurs, the higher disk temperature causes the mark on the disk to be larger than if the bias power were lower. For the 8T lone, the marks are isolated so that the amplitude grows with bias power at a given write power. The marks for the 3T tone, however, are spaced much closer and the tighter spacing causes interference hetween marks. As bias power increases, the marks get larger, thereby increasing the interference. When the write power is low enough, the marks are small and there is little interference. An increase in bias power would cause an increase in amplitude in the manner described above for the 8T tone. At higher write powers, the marks become much larger and inter-symbol interference dominates and causes the amplitude to drop.

Jitter and amplifude are clearly dependent on bias power. The minimum achievable jitter increases as bias power increases (see Figures 3 and 4). Also, the sensilivity to write power increases as bias power increases. A small change in write power for the $2 \mathrm{~mW}$ case causes more degradation in performance than the same percentage change at a $0.5 \mathrm{~mW}$ bias power. Because our system is strictly media noise dominaled al the data rates evaluated, minimum jitter scales inversely with velocity. The 
Finkelstein and Wagner: Write Performance and the Influence of Bias Power

increase in minimum jitter (the jitter at the optimum write power) with an increase in bias power is because of changes in thermal gradients during write. At low bias powers, the thermal gradient is sharp, which helps to speed cooling. When cooling occurs rapidly, there is less variation in mark size and the jitter decreases. The authors speculated that pattern dependent peak shif would decrease as bias power increased, but no evidence was found to support the speculation.

CONCLUSIONS - Optimum write power is a strong function of bias power. Each increase of $0.5 \mathrm{~mW}$ in bias power reduces the required write power between 1 and $1.5 \mathrm{~mW}$; however, lower bias powers produce less jitter. Because of the availability of high power lasers needed to write at low bias powers and the reduction in jitter previously mentioned, the lowest possible bias power consistent with servo performance should be used. The increased margins will translate to higher data reliability. The dependence of write power and jitter on bias power was expected, but the independence of resolution, amplitude, and peak shift at optimum write power, was unexpected.

Figure 1

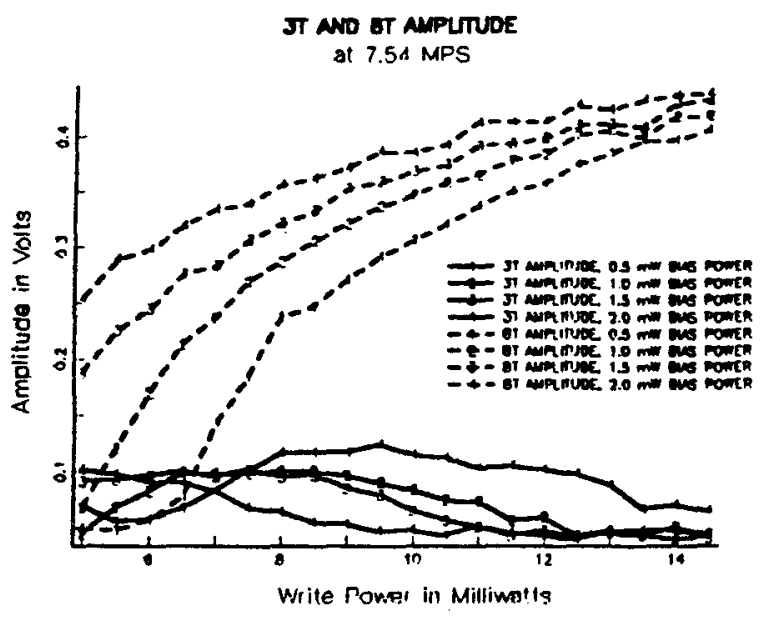

Finue 3

IT ANO ET ATIER at $7.54 \mathrm{MFS}$

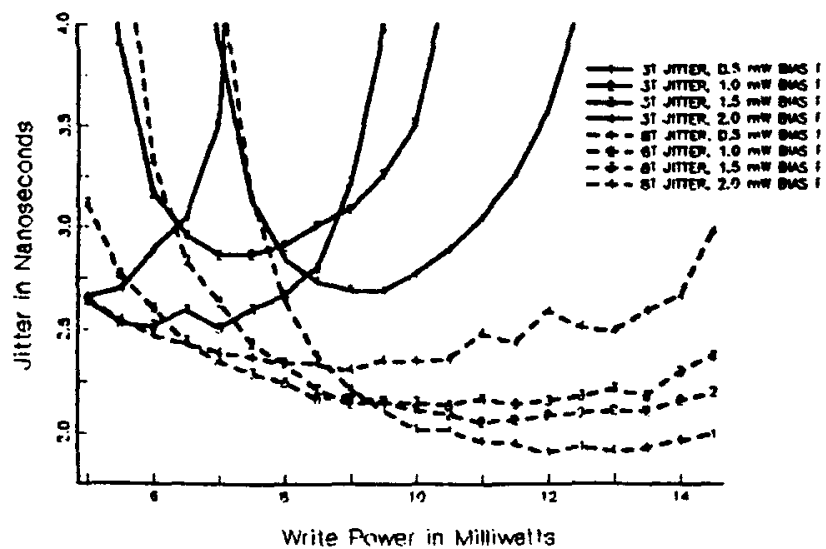

Figure 1. 3T and 8T Amplitude
Figurs 2

3T ANO ET AMPUTUDE

31 14.61 A.MPS

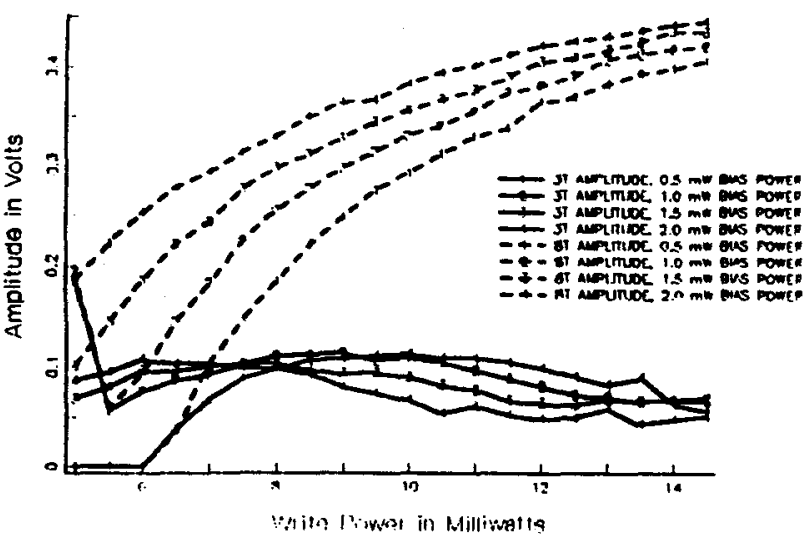

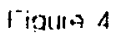

JT ANO OT JTIER ? 11.611 .45

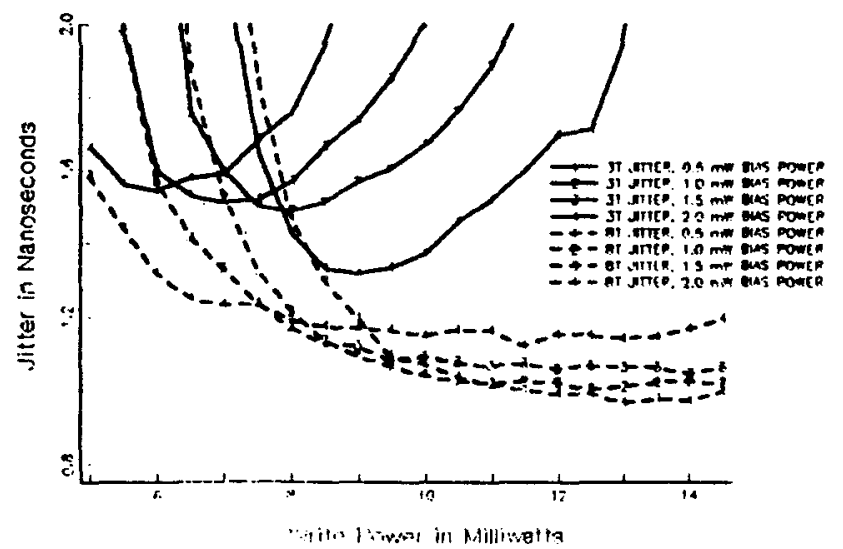

